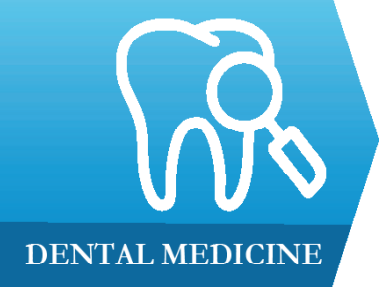

DENTAL MEDICINE

Sri Aurobindo College of Dentistry, Indore, India

DOI: $10.15386 / \mathrm{mpr}-1467$

Manuscript received: 08.09.2019

Received in revised form: 29.05 .2020

Accepted: 15.06.2020

Address for correspondence:

drshaleenvyas@gmail.com

This work is licensed under a Creative Commons Attribution-NonCommercialNoDerivatives 4.0 International License

\section{Smartphone assisted oral health data recording - an android based software application development}

Sandesh Nagarajappa, Shaleen Vyas

\begin{abstract}
Background. Smartphone compared to the traditional pen-paper method could enhance oral health data recording procedure by reducing the cost of data collection, risk of data loss, early detection of errors and reducing data entry time. The present research developed a mobile/tablet-based software application to capture oral health data and test its adaptability and operations in oral health surveys.

Methods. A comparative cross-sectional study was conducted among the general population of Sanwer town, Indore district. The initial testing of the application was done on 120 individuals. A random sampling (lottery method) followed by a systematic sampling strategy was employed to select 120 households. A "one per household" design was implemented for the survey. The initial oral health data collection was done using mobile-assisted software application followed by a second examination scheduled after 15 days on the same participants using the conventional Pen-paper method to collect oral health data.

Results. Six Investigator Recorder (IR) teams conducted the oral health data collection. Data collection through Smartphone-based application displayed less meantime (3.57 minutes) in comparison to pen-paper method (4.87 minutes) $(\mathrm{p} \leq 0.001)$. Survey team response showed the majority of investigators having strong agreement on user satisfaction and speed of data entry using software application.

Conclusion. The initial testing of mobile-assisted recording system (MARS) efficiently captured oral health data among the general population with wide variations in oral disease level. The application facilitated minimal or no wastage of paper and had a high level of user-satisfaction, accuracy, speed of entry and low potential for any data loss.
\end{abstract}

Keywords: oral health, software validation, electronic data collection, technology assessment, survey research, mobile health

\section{Background and aim}

Mobile technology is a powerful media for providing individual-level support to health care providers. In recent years, mobile technology has been used in medicine and health care for retrieving patient information, clinical data collection, and health survey [1]. The method of data collection is critical to health research and often is a predisposing factor determining the cost and efficacy of a research project. It also has a definitive impact on time, accuracy and other parameters related to research [2].

The use of handheld devices for data collection have been previously reported in clinical settings and numerous research in areas $[3,4]$. Compared to traditional penpaper based system, the handheld devices are said to have advantage with lower risk of data loss, early detection of systematic data errors, high user acceptance, less time for data entry and reduced cost of data collection in large surveys [5-7].

An ideal data-collection tool should ensure wider application, be inexpensive 
and easy to use with minimal training on technical aspects and methods of data entry. An application such as OpenData Kit and Epi-Collect have spurred the use of touchscreen smartphones and tablet computers for data collection in public health research [8-10].

In health research, several studies have been conducted comparing the pen-paper method with various electronic devices such as personal digital assistants (PDA, Palmtop computer), tablet computers and smartphones $[11,12]$. Handheld electronic devices have the potential to produce similar data accuracy compared to paper-based methods along with a considerable reduction of time. They also hold the promise to reduce research associated costs on large scale surveys $[13,14]$. The application of similar technology in oral health research is lacking. The rationale behind conducting this study was to incorporate the use of the mobile phone-based android application in oral health surveys. Hence we hypothesized that compared to a paperbased system, the use of mobile/tablet software application for oral health data collection would be well accepted by the user thereby reducing at least $20-30$ percent of data entry errors and time required in different survey conditions.

Besides, it is important to determine whether different data collection/entry methods (mobile assisted or pen-paper) influence the content of data collected and also whether oral health care providers would readily adopt this digital platform. Thus, the present study objectified to develop and test an android software application for appropriate data entry, user satisfaction rates, and cost-benefits in an oral health survey.

\section{Methods \\ Development of Mobile Assisted Recording System (MARS)}

The application system for recording oral health data using a smartphone device was built on an android platform (Figure 1). The application was named "MARS". In Oral health research, much of data collection is through the use of various 'indices' that quantify oral health condition in terms of numerical values. The World Health Organization (WHO) Oral Health Survey manual also proposes a pen-paper based 'Oral health assessment form' for a comprehensive recording of the oral health status of adults/children [15]. In the Indian scenario, almost all oral health surveys and clinical studies employ pen-paper recording. Consequently, the activity behind developing MARS was to disentangle the oral health information recording with extreme exactness, client fulfillment, and minimum error rates.

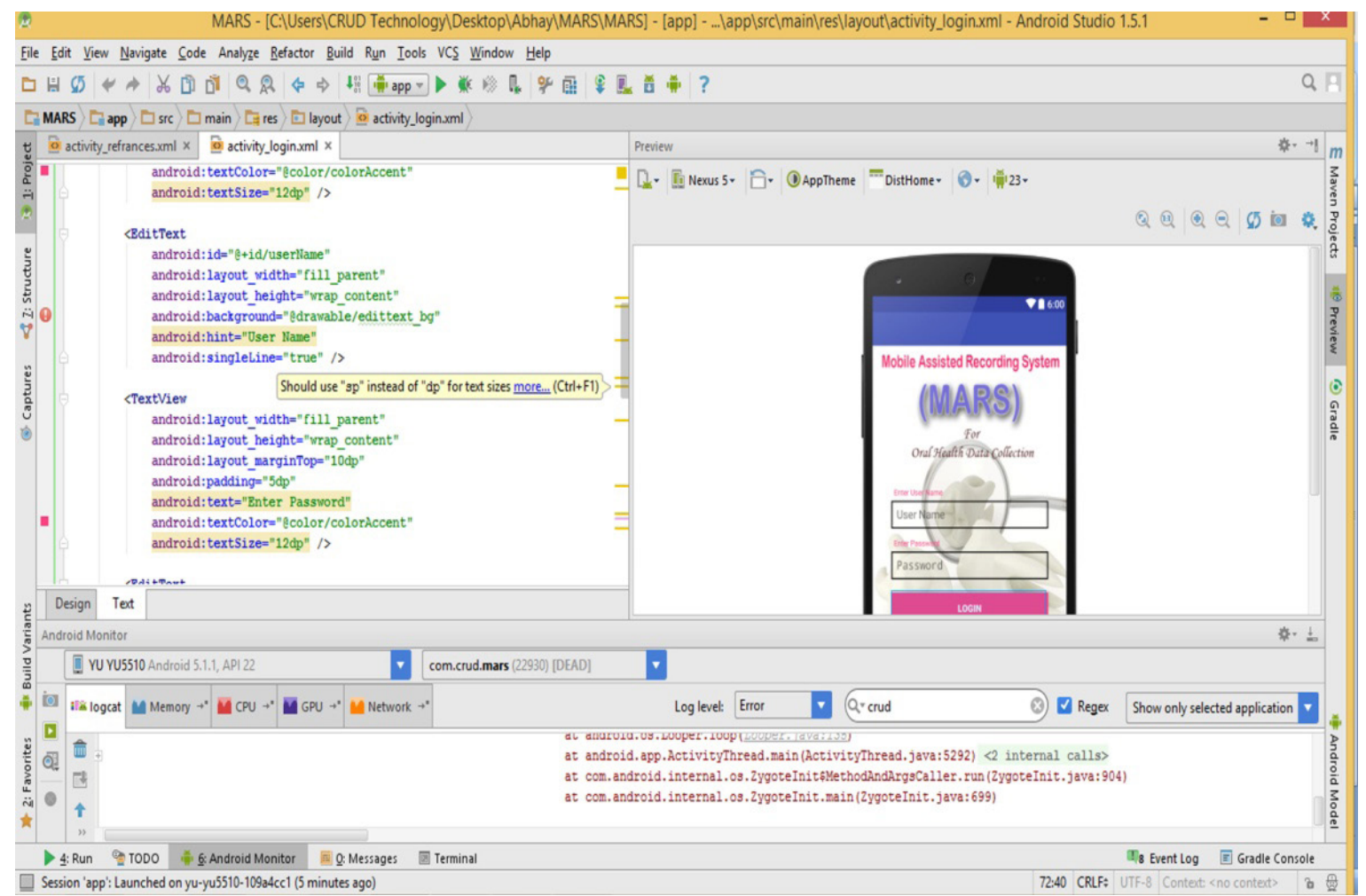

Figure 1. Building MARS application using android platform. 
MARS consisted of unique features like a user-ID and password for security issues on the confidentiality of the data collected. The area under survey could be documented either using a numerical code or area name in the survey-code entry box for future reference. The application employs indices to record oral health data on dental caries experience, periodontal disease, oral hygiene status, gingival status, plaque score, and dental fluorosis status. The data on caries experience was collected using DMFT/DMFS index [16]. Individual boxes to capture data on total decayed, totally missed and total filled teeth or surface were created. The cumulative score was automatically generated in a separate box.

For the recording of dental fluorosis, two entities were created. The first dialogue box represented the arch (upper/lower) and the second box denoted the teeth affected. Periodontal status of participants was assessed through the community periodontal index (CPI) and loss of attachment (LOA) [17]. Six dialogue boxes each representing the index teeth were formulated. Two individual dialogue boxes were framed to input overall score of community periodontal index and loss of attachment respectively. The android application functions on a sequential numbering system. Hence, code " $X$ " and code "9" were coded as " 5 " and " 6 " respectively in both CPI and LOA categories.

For recording the oral hygiene status, separate debris and calculus recording boxes were provided in the application [18]. A special feature incorporated was an inbuilt numerical calculator. The total scores for debris and calculus index were automatically generated after addition of individual scores and divided by the number of teeth assessed. Besides the individual calculations, debris and calculus scores were also put to automatic generation of OHI-S scores and interpretation.

Data regarding plaque scores were collected using the plaque index [19]. Both tooth-wise, as well as overall plaque scores, could be calculated by adding individual values and dividing by the number of surfaces/index teeth examined. A final dialogue box with a manual entry was provided for incorporating any additional information about participant's oral health. To avoid any missing entries, a final submission button ensured completeness of all entries. A pop-up window was created containing information to be selected for respective indices. The "application" was so designed that operators can click on relevant option to enter information into dialogue boxes. List of references for all indices used along with a sign-in disclaimer was provided after log-in.

Additional options included data generation, deletion of any entry, references, and an exit button. The application was developed with a specific feature of generating all coded/non-coded information directly into "Excel (2007) (Microsoft Corp, Redmond, Washington, USA)". The information so generated was designed to be saved in a folder subsequently generated at the time of "application" installation. Each entry was planned to be saved in the folder according to date of entry. The generated Excel sheet could be directly used for the statistical analysis of the data collected.

Pen-paper format used in the study was exactly similar to the developed mobile application. A supplement paper was provided as a reminder for various coding. The data using Pen-paper method was collected 15 days after initial data collection using MARS.

The participants were revisited through the household's identification number and contact numbers obtained through them during the initial visit. The Penpaper survey team was supposed to manually calculate the recorded findings. The investigators were free to either write the full finding or code them.

The developed application was pilot tested for operations and functionalities on five android mobile and Tablet devices. To ensure the completeness, different test cases or test scenarios were created. Any differences between actual \& expected results were reported as defects. Once the developer fixed the reported defects, a retest was employed to assure success. A minimum of 20 participants belonging to the age range of $15-75$ years was randomly chosen for the pilot study to check the feasibility of mobile-based data collection. A rough estimate of the time required for data collection and entry was documented. Based on the results and feedback of pilot testing, iterations were made in the final program model.

\section{Field-testing}

The present study employed 12 investigators with the background of oral health data collection through surveys. All these investigators were trained for examining and recording of oral health data. They were divided into six investigator-recorder teams. The study tools used in the present study included MARS application and penpaper proforma. The pen-paper proforma was exactly similar to MARS.

The investigators and recorders participating in the field-testing survey were trained about technical and operating aspects of using the application. A calibration session for mobile-based and paper-based recording was conducted with the help of a subject expert and the developer team of MARS. Calibration exercise was carried out a week after the training session and continued till all investigators matched gold standards of a subject expert. 


\section{Survey utilized}

A cross-sectional oral health survey was conducted among the general population of Sanwer town, Indore district. A convenient sample of 120 individuals was selected through a household survey. Individuals belonging to the age group 15-75 years, residing in the same area past 5 years, with at least 21 functional teeth (WHO, 1991) and providing consent were included in the study [20]. Individuals with severe debilitating health conditions or medically compromised state and any condition limiting oral health examination were excluded. Ethical clearance was obtained from the Institutional review board.

In the first survey, the selected sample was examined according to the WHO Oral health survey procedure and the oral health data was recorded using MARS. All members of IR-teams examined and recorded an equal number of study sample each $(n=10)$. In the followup survey scheduled three weeks after, the same study sample was revisited with the help of house number and other contact details. Oral health data was again recorded by IR teams using a Pen-paper format. In every visit, the outcome measure was data entry time and problems experienced during data collection. The field coordinator recorded outcome measure using a digital watch.

After completion of the survey using both methods, data were generated into "Excel 2007 (Microsoft Corp, Redmond, Washington, USA). The time required for data entry and the excel sheet generation with both pen-paper format and MARS was assessed. The data entry was crosschecked for any entry errors with pen-paper format. The generated excel sheet of both data collection methods was also matched and any data entry errors were identified and recorded.

In the end, the IR-team were requested to complete a self-administered questionnaire rated on a five-point Likert scale to assess other outcome measures like user satisfaction, user-friendliness, accuracy, speed of entry and potential for data loss using both methods of data collection.

\section{Statistical analysis}

Data collected on the outcome measure of the study was analyzed using Statistical Package for Social Sciences, SPSS Version 20.0. (2011), (IBM SPSS Statistics for Windows, Armonk, NY: IBM Corp.). The level of significance was set at $5 \%$ and $p$-value $<0.05$ was considered statistically significant. Descriptive statistics were used to find the frequencies, mean and standard deviation of outcome variables. Mann-Whitney U-test was employed to assess the mean difference in time and other parameters between the two groups.

\section{Results}

The results of the present study are based on a field survey conducted to collect oral health data by 12 oral health professionals using MARS and pen-paper method. The investigators' profile is presented in table I.

Table I. Details of the oral health professional team with individuals profile in relation to demographic variables.

\begin{tabular}{llc}
\multicolumn{3}{c}{ Oral Health Professionals-team profile $(\mathbf{N}=\mathbf{1 2})$} \\
\multirow{2}{*}{ Age } & Mean & 25.58 years \\
& Range & $22-30$ years \\
\multirow{2}{*}{ Gender } & Male; $\mathrm{n}(\%)$ & $6(50 \%)$ \\
& Female; $\mathrm{n}(\%)$ & $6(50 \%)$ \\
\multirow{5}{*}{ Education } & Graduation; $\mathrm{n}(\%)$ & $6(50 \%)$ \\
& Post graduation; $\mathrm{n}$ & $6(50 \%)$
\end{tabular}

The study participants on whom the survey was conducted belonged to the age group of 15-75 years (Table II).

Table II. Distribution of study subjects in relation to sociodemographic variables.

\begin{tabular}{|c|c|c|}
\hline Variables & Categories & $\begin{array}{c}\text { No. of } \\
\text { participants } \\
\text { n }(\%)\end{array}$ \\
\hline \multirow{4}{*}{ Age } & $15-25$ years & $52(43.3)$ \\
\hline & $26-35$ years & $28(23.3)$ \\
\hline & $36-45$ years & $30(25)$ \\
\hline & 46 years and above & $10(8.4)$ \\
\hline \multirow{2}{*}{ Gender } & Males & $62(51.7)$ \\
\hline & Females & $58(48.3)$ \\
\hline \multirow{5}{*}{ Education } & Uneducated & $30(25)$ \\
\hline & Pre-schooling & $4(3.3)$ \\
\hline & Higher-schooling & $26(21.7)$ \\
\hline & Under graduate & $38(31.7)$ \\
\hline & Post graduate & $22(18.3)$ \\
\hline \multirow{6}{*}{ Occupation } & Professional & $32(26.7)$ \\
\hline & Intermediate & $14(11.7)$ \\
\hline & Skilled non-manual & $12(10)$ \\
\hline & Skilled manual & $18(15)$ \\
\hline & Partially skilled & $10(8.4)$ \\
\hline & Unskilled & $34(28.3)$ \\
\hline \multirow{4}{*}{ Religion } & Hinduism & $110(91.7)$ \\
\hline & Muslim & $2(1.7)$ \\
\hline & Sikh & $6(5)$ \\
\hline & others & $2(1.7)$ \\
\hline \multirow{4}{*}{$\begin{array}{l}\text { Per capita } \\
\text { monthly } \\
\text { income }\end{array}$} & Lower $(0-10,000)$ & $64(53.3)$ \\
\hline & Lower middle $(10,000-20,000)$ & $24(20)$ \\
\hline & Upper middle $(20,000-30,000)$ & $24(20)$ \\
\hline & Upper $(30,000-50,000)$ & $8(6.7)$ \\
\hline
\end{tabular}

The time required for oral health data collection by both methods was recorded and compared (Table III). 
Table III. Comparison of different data collection methods (MARS/pen-paper) in relation to the time required for recording oral health related data.

\begin{tabular}{l|c|cc}
\multirow{2}{*}{$\begin{array}{l}\text { Variable } \\
\text { (participants examined) }\end{array}$} & $\begin{array}{c}\text { MaRS (time) } \\
\text { mean } \pm \text { SD } \\
\text { (Min.-Max.) }\end{array}$ & $\begin{array}{c}\text { Pen-paper method (time) } \\
\text { mean } \pm \text { SD } \\
\text { (Min.-Max.) }\end{array}$ & p-value \\
\cline { 2 - 4 } & $3.57 \pm 0.57(2.59-5.10)$ & $4.87 \pm 0.63(4.00-6.00)$ & $\mathbf{0 . 0 0 1}$ (HS) \\
Total Time $(\mathrm{N}=120)$ & $3.45 \pm 0.27(3.00-4.30)$ & $4.78 \pm 0.53(4.00-5.50)$ & $\mathbf{0 . 0 0 1}$ (HS) \\
Investigator 1 $(\mathrm{n}=10)$ & $3.57 \pm 0.54(3.00-4.50)$ & $4.92 \pm 0.49(4.00-6.00)$ & $\mathbf{0 . 0 0 1}$ (HS) \\
Investigator 2 $(\mathrm{n}=10)$ & $3.40 \pm 0.43(2.59-4.56)$ & $4.77 \pm 0.57(4.00-6.00)$ & $\mathbf{0 . 0 0 2}$ (HS) \\
Investigator 3 $(\mathrm{n}=10)$ & $3.86 \pm 0.66(3.00-5.10)$ & $4.94 \pm 0.23(4.05-6.00)$ & $\mathbf{0 . 0 0 6}$ (HS) \\
Investigator 4 $(\mathrm{n}=10)$ & $3.76 \pm 0.46(3.05-5.04)$ & $4.84 \pm 0.13(4.15-6.00)$ & $\mathbf{0 . 0 0 1}$ (HS) \\
Investigator 5 $(\mathrm{n}=10)$ & $3.46 \pm 0.86(3.20-5.10)$ & $4.64 \pm 0.28(4.00-6.00)$ & $\mathbf{0 . 0 0 1}$ (HS) \\
Investigator 6 $(\mathrm{n}=10)$ & $3.45 \pm 0.27(3.00-4.30)$ & $4.78 \pm 0.53(4.00-5.50)$ & $\mathbf{0 . 0 0 1}$ (HS) \\
Investigator 7 $(\mathrm{n}=10)$ & $3.57 \pm 0.54(3.00-4.50)$ & $4.92 \pm 0.49(4.00-6.00)$ & $\mathbf{0 . 0 0 1}$ (HS) \\
Investigator 8 $(\mathrm{n}=10)$ & $3.40 \pm 0.43(2.59-4.56)$ & $4.77 \pm 0.57(4.00-6.00)$ & $\mathbf{0 . 0 0 2}$ (HS) \\
Investigator 9 $(\mathrm{n}=10)$ & $3.86 \pm 0.66(3.00-5.10)$ & $4.94 \pm 0.23(4.05-6.00)$ & $\mathbf{0 . 0 0 6}$ (HS) \\
Investigator 10 $(\mathrm{n}=10)$ & $3.76 \pm 0.46(3.05-5.04)$ & $4.84 \pm 0.13(4.15-6.00)$ & $\mathbf{0 . 0 0 1}$ (HS) \\
Investigator 11 $(\mathrm{n}=10)$ & $3.46 \pm 0.86(3.20-5.10)$ & $4.64 \pm 0.28(4.00-6.00)$ & $\mathbf{0 . 0 0 1}$ (HS)
\end{tabular}

$(H S)=$ Highly significant $(p \leq 0.001) ;$ Test: Mann Whitney U-test

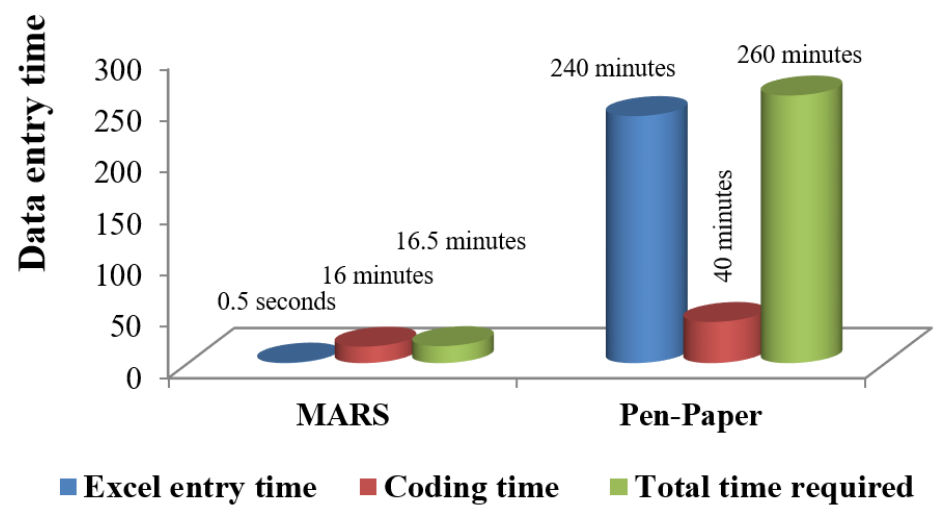

Graph 1. Time required for data entry into microsoft excel sheet through different methods.

The MARS required significantly lower meantime (3.57 minutes) in comparison to Pen-paper method (4.87 minutes) [Mann-Whitney $U$ test, $p<0.001$ ]. The timerange limit of data collection for all 12 investigators using MARS (2.59 - 5.10 minutes) was significantly lower in comparison to Pen-paper method (4.00-6.00 minutes).

No significant mean difference was observed among the two different groups concerning oral health scores like debris score, calculus score, OHI-S score, plaque score, and CPI-LOA scores. The depreciation in clinical scores in the pen-paper group was attributed to entry and calculation errors committed during data collection (Table IV).
Table IV. Comparison among different data collection methods (MARS/Pen-paper) in relation to other oral health scores recorded.

\begin{tabular}{c|c|c|c}
\multirow{2}{*}{ Variable } & \multicolumn{2}{|c|}{ Data collection methods } & \multirow{2}{*}{ p-value } \\
\cline { 2 - 3 } & MARS & $\begin{array}{c}\text { Pen-paper } \\
\text { method }\end{array}$ & \\
\hline Debris score * & $1.65 \pm 0.86$ & $1.68 \pm 0.75$ & 0.81 \\
Calculus score * & $1.71 \pm 1.07$ & $1.69 \pm 1.15$ & 0.82 \\
OHI-S score * & $1.09 \pm 0.76$ & $1.07 \pm 0.80$ & 0.57 \\
Plaque score * & $1.46 \pm 0.80$ & $1.23 \pm 0.72$ & 0.11 \\
CPI scores * & $1.86 \pm 0.87$ & $1.83 \pm 0.90$ & 0.72 \\
LOA scores * & $0.20 \pm 0.48$ & $0.16 \pm 0.41$ & 0.64 \\
Error rate \# & $2.50 \%$ & $9.33 \%$ & $\mathbf{0 . 0 4}$
\end{tabular}

* Scores presented as mean \pm SD; Test: Mann Whitney U-test; \# Error rates expressed as percentage error of committing two or more errors per data entry. 
Table V. Investigators' response on user-satisfaction, user-friendliness, speeds of entry, accuracy and potential for data loss using MARS application in comparison to Pen-paper method.

\begin{tabular}{cc|c|c|c|c|c|}
\multirow{2}{*}{ Variables } & \multicolumn{5}{c|}{ Responses recorded on five point Likert scale, n (\%) } \\
\cline { 3 - 7 } & & Definitely yes & Yes & Neutral/don't know & No & Definitely no \\
\cline { 3 - 7 } User & MARS & $9(75 \%)$ & $3(25 \%)$ & $0(0 \%)$ & $0(0 \%)$ & $0(0 \%)$ \\
satisfaction & Pen-paper & $0(0 \%)$ & $0(0 \%)$ & $3(25 \%)$ & $3(25 \%)$ & $6(50 \%)$ \\
User & MARS & $6(50 \%)$ & $3(25 \%)$ & $3(25 \%)$ & $0(0 \%)$ & $0(0 \%)$ \\
friendly & Pen-paper & $0(0 \%)$ & $0(0 \%)$ & $3(25 \%)$ & $9(75 \%)$ & $0(0 \%)$ \\
Accuracy & MARS & $6(50 \%)$ & $6(50 \%)$ & $0(0 \%)$ & $0(0 \%)$ & $0(0 \%)$ \\
& Pen-paper & $0(0 \%)$ & $0(0 \%)$ & $3(25 \%)$ & $9(75 \%)$ & $0(0 \%)$ \\
Speed of entry & MARS & $12(100 \%)$ & $0(0 \%)$ & $0(0 \%)$ & $0(0 \%)$ & $0(0 \%)$ \\
& Pen-paper & $0(0 \%)$ & $0(0 \%)$ & $0(0 \%)$ & $6(50 \%)$ & $6(50 \%)$ \\
Data loss & MARS & $0(0 \%)$ & $0(0 \%)$ & $3(25 \%)$ & $9(75 \%)$ & $0(0 \%)$ \\
& Pen-paper & $3(25 \%)$ & $3(25 \%)$ & $6(50 \%)$ & $0(0 \%)$ & $0(0 \%)$
\end{tabular}

The MARS-oral health application was designed to generate the output of survey in "Excel 2007 (Microsoft Corp, Redmond, Washington, USA)". Hence the data entry and coding time required with the application was relatively less (16.5 minutes) (Graph 1). Comparatively, the data collected by pen-paper method was to be entered manually into the system and coded. So the total time required for data entry and coding of all 120 participants using the conventional method was 260 minutes.

The user satisfaction and views of investigators on the speed of entry, accuracy, and potential data loss were assessed through a self-administered questionnaire. The response showed $75 \%$ of investigators with a strong agreement to user satisfaction with MARS in data collection/entry. The automatic generation of output in the desired format enabled all investigators to respond to a strong agreement $(100 \%)$ on the speed of data entry. Majority of investigators (75\%) considered the application to be extremely user-friendly in comparison to traditional pen-paper method. The study results showed $50 \%$ of investigators with a strong predilection for potential loss of data using traditional Pen-paper method (Table V).

Besides, subjective assessment on the utilization of MARS exhibited the least wastage of paper hence advancing natural amicable green activity.

\section{Discussion}

Although p aper-based data collection has been the standard method for decades, errors are still frequent, storage costs are prohibitive, and costs of double data entry are high $[1,2,5]$. The present comparative cross-sectional study was carried out to develop and test the efficiency of MARS in an oral health survey. The study highlights the adaptability and user satisfaction among oral health professionals in recording oral health data using a mobileassisted recording system.

The present study differed from the literature of similar projects bychoosing hospital-based population instead of the general population $[21,22]$. Tomlinson et al., (2009) utilized a similar study design to assess the use of mobile phones as a data collection tool among the general population [23]. The present study employed MARS to collect oral health data using the investigatoradministered method. The prime focus of our study was to gather responses of IR-team on usability and applicability of MARS in contrast to evaluating participant's response $[8,21,22]$.

The relatively small sample size was selected considering the initial pilot-phase of testing MARS. Although, King et al., (2013) conducted a large scale Trachoma survey using electronic Android-based data collection technology in Ethiopia and established its accuracy and preference by recorders over standard paperbased questionnaires [22].

Identical investigator-recorder profile and characteristics excluded the bias due to demographic variations. The results of the present study suggest that cumulative time taken to collect oral health-related data using mobile version was relatively less in comparison to traditional pen-paper method, thus increasing the productivity by saving time. The present study overcomes the deficiency in evaluating cumulative as well as individual investigators time in data collection, coding and data entry respectively. The automated output generation in the desired format reduced the bulk amount of time and man-hours spent in data entry procedure. Van Den Kerkhof EG (2005) reported similar findings with only median cumulative time to complete Pre-Admission Adult Anesthetic Questionnaires (PAAQ) [24-26]. MARS - oral health application demonstrated a lower error rate in data recording in comparison to pen-paper method. The observed error rates were subjected to individual investigators potentiality in the recording. The error rate of committing two or more errors per data entry in Pen-paper 
group was observed among 9.33\% cases. King et al., (2013) also reported a significant difference in the error rates in data entry in between paperless and pen-paper methods [22]. The percentage of at least one blank field in census record (age, sex, availability) in paper-based method was $1.7 \%$ in comparison to $1.5 \%$ in the paperless method of data collection. To overcome the limitations of previous literature concerning data entry errors, we assessed the percentage omission rates also for additional informatory data entered by the two methods. The use of mobile assisted technology yielded cent-percent response rate on accuracy of data collected. The accuracy was imparted to the MARS application by creating text field filters and strict data submission protocol, ensuring correct entries.

The mobile-assisted technology "MARS" yielded a $100 \%$ response rate by the oral health professionals on the accuracy of data collected. Besides, they reported a high degree of user-satisfaction through MARS. The current scenario on mobile usage enabled us to develop and test MARS for user-satisfaction and user-friendliness in contrast to the conventional method. Previous studies reported dominance of traditional Pen-paper method for data collection as use of computers was cumbersome in a clinical setting and also the health care professionals were more adapted to the use of conventional Pen-paper method to enter data $[26,27]$.

The confidence of investigators on the ability of MARS to retain data in the present study is attributed to back-up file generation and data support system incorporated within the application. Galliher et al., (2008) conducted a study on data collection outcomes comparing paper forms with hand-held tablets (PDA) forms in an office-based patient survey. The results from this study indicated that although handheld computers produced more complete data than a paper method, they were not superior because of a large amount of missing data due to technical difficulties with handheld computers or loss or theft [6].

A high initial start-up cost was incurred in developing and testing MARS in contrast to pen-paper method. In the present study, cost-effectiveness of mobile operated the system was evaluated on the basis of man-hours required in conducting each operational phase including data collection, coding, and data entry. The depreciation in man-hours required demonstrates the effectiveness of mobile-assisted recording system in large surveys.

The mobile-assisted recording system was employed among the age range 15-75 years, thus limiting the testing below 15 years of age group. Relative small sample size limits the generalizability of the results. There may be a probability of investigators bias. Nevertheless, initial testing of MARS efficiently captured oral health data among the general population with wide variations in oral disease level. Compared to Pen-paper method, it reduced the time taken to record oral health data and efforts on double data entry. The developed application has a high level of user-satisfaction, accuracy, speed of entry and low potential for any data loss.

The mobile assisted recording system was employed among population of age range 15-75 years, thus limiting the testing of MARS "app" among subjects below 15 years of age. Despite the attempts made to avoid bias in our study, there may be probability of investigators bias while collecting and entering the oral health related data. A lack of calculation capability of "app" in recording oral hygiene index among cases with missing index tooth was reported by the investigators. The battery life of smart phones could be an issue in larger oral health surveys in rural and remote areas with lack of electricity. The generalizability of the results from the present study is limited as the sample size is relatively small. Larger surveys using MARS application is recommended to analyze the cost-effectiveness of application.

\section{Conclusion}

The initial testing of mobile assisted recording system (MARS) efficiently captured oral health data among general population with wide variations in oral disease level. The developed application met the idea requirements of being considered as a comprehensive "app" to record oral health data like applicability, usability and productivity. MARS application reduced the time taken to record oral health data along with reduced efforts on double data entry into excel format. The application facilitated minimal or no wastage of paper thus contributing to the environmental friendly - Green advantage. The developed application has high level of user-satisfaction, accuracy, speed of entry and low potential for any data loss.

The present study penetrates into the exciting new area of work related to the use of mobile phone based technology to help oral health professionals to manage the oral health of people. Effective utilization of MARS "app" can facilitate easy monitoring and transfer of data through electronic media in large-scale surveys. It also projects the adaptability of utilizing MARS "app" in field researches in developing nations and areas with no internet accessibility.

\section{Acknowledgements}

The study acknowledges the investigator-recorder teams for their patience throughout the study. We also acknowledge the study participants who cooperated to provide their oral health data and the software developing team for providing anytime assistance on technical issues in MARS.

\section{References}

1. Curl M, Robinson D. Hand-held computers in clinical audit: a comparison with established paper and pencil methods. Int J Health Care Qual Assur. 1994;7:16-20. 
2. McBride JS, Anderson RT, Bahnson JL. Using a hand-held computer to collect data in an orthopedic outpatient clinic: A randomized trial of two survey methods. Med Care. 1999;37:647-651.

3. Fjeldsoe BS, Marshall AL, Miller YD. Behavior change interventions delivered by mobile telephone short-message service. Am J Prev Med. 2009;36:165-173.

4. Lim MS, Hocking JS, Hellard ME, Aitken CK. SMS STI: A review of the uses of mobile phone text messaging in sexual health. Int J STD AIDS. 2008;19:287-290.

5. Missinou MA, Olola $\mathrm{CH}$, Issifou $\mathrm{S}$, Matsiegui $\mathrm{PB}$, Adegnika AA, Borrmann S, et al. Short report: piloting paperless data entry for clinical research in Africa. Am J Trop Med Hyg. 2005;72:301-303.

6. Galliher JM, Stewart TV, Pathak PK, Werner JJ, Dickinson LM, Hickner JM. Data collection outcomes comparing paper forms with PDA forms in an office-based patient survey. Ann Fam Med. 2008;6:154-160.

7. Burton C, Weller D, Sharpe M. Are electronic diaries useful for symptoms research? A systematic review. J Psychosom Res. 2007;62:553-561.

8. Lane SJ, Heddle NM, Arnold E, Walker I. A review of randomized controlled trials comparing the effectiveness of hand held computers with paper methods for data collection. BMC Med Inform Decis Mak. 2006;6:23.

9. Free C, Phillips G, Galli L, Watson L, Felix L, Edwards $\mathrm{P}$, et al. The Effectiveness of Mobile-Health TechnologyBased Health Behaviour Change or Disease Management Interventions for Health Care Consumers: A Systematic Review. PLoS Med. 2013;10:e1001362.

10. Walther B, Hossin S, Townend J, Abernethy N, Parker D, Jeffries D. Comparison of electronic data capture (EDC) with the standard data capture method for clinical trial data. PLoS One. 2011;6:e25348.

11. Prinja S, Nimesh R, Gupta A, Bahuguna P, Thakur JS, Gupta $M$, et al. Impact assessment and cost-effectiveness of m-health application used by community health workers for maternal, newborn and child health care services in rural Uttar Pradesh, India: a study protocol. Glob Health Action. 2016;9:31473.

12. Watts S, Mackenzie A, Thomas C, Griskaitis A, Mewton L, Williams A, et al. CBT for depression: a pilot RCT comparing mobile phone vs. computer. BMC Psychiatry. 2013;13:49.

13. Denny SJ, Milfont LT, Utter J, Robinson EM, Ameratunga SN, Merry SN, et al. Hand-held internet tablets for schoolbased data collection. BMC Res Notes. 2008;1:52.
14. Free C, Phillips G, Felix L, Galli L, Patel V, Edwards P. The effectiveness of M-health technologies for improving health and health services: a systematic review protocol. BMC Res Notes. 2010;3:250.

15. World Health Organization. Oral health assessment form for adults. Oral Health Survey Basic Methods, Fifth edition 2013;5, p. 6-85.

16. Klein $\mathrm{H}$, Palmer CE. The dental problem of elementary school children. The Milbank memorial fund quarterly 1938;16:267-286.

17. Cutress TW, Ainamo J, Sardo-Infirri J. The community periodontal index and treatment needs (CPITN) procedure for population groups and individuals. Int Dent J. 1987;37:222228.

18. Greene JC, Vermillion JR. The simplified oral hygiene index. J Am Dent Assoc. 1964;68:7-13.

19. Loe H, Silness J. Periodontal disease in pregnancy. I. Prevalence and severity. Acta Odontol Scand. 1963;21:533-551.

20. Leopold CT, Mander C, Utting C, Watkins K, Rock WP. The World Health Organization goals for oral health: a progress report. Community Dent Health. 1991;8:245-251.

21. DeRenzi B, Borriello G, Jackson J, Kumar VS, Parikh TS, Virk P, et al. Mobile phone tools for field-based health care workers in low-income countries. Mt Sinai J Med. 2011;78:406-418.

22. King JD, Buolamwini J, Cromwell EA, Panfel A, Teferi T, Zerihun $\mathrm{M}$, et al. A novel electronic data collection system for large-scale surveys of neglected tropical diseases. PLoS One. 2013;8:e74570.

23. Tomlinson M, Solomon W, Singh Y, Doherty T, Chopra M, Ijumba $\mathrm{P}$, et al. The use of mobile phones as a data collection tool: a report from a household survey in South Africa. BMC Med Inform Decis Mak. 2009;9:51.

24. VanDenKerkhof EG, Goldstein DH, Blaine WC, Rimmer MJ. A comparison of paper with electronic patient-completed questionnaires in a preoperative clinic. Anesth Analg. 2005;101:1075-1080.

25. Draijer G, Kalfs N, Perdok J. Global Positioning System as Data Collection Method for Travel Research. Transportation Research Record. 2000;1719:147-153.

26. Bushnell DM, Martin ML, Parasuraman B. Electronic versus paper questionnaire: a further comparison in persons with asthma. J Asthma. 2003;40:751-762.

27. Wilcox AB, Gallagher KD, Boden-Albala B, Bakken SR. Research data collection methods: from paper to tablet computers. Med Care. 2012;50 Suppl:S68-S73. 